\title{
Effect of chronic undernutrition on body mass and mechanical bone quality under normoxic and altitude hypoxic conditions
}

\author{
Christian Lezon, Clarisa Bozzini, Alan Agûero Romero, Patricia Pinto, Graciela Champin, Rosa M. Alippi, \\ Patricia Boyer and Carlos E. Bozzini* \\ Department of Physiology, Faculty of Odontology, University of Buenos Aires, Buenos Aires C1122 AAH, Argentina
}

(Submitted 14 September 2015 - Final revision received 17 December 2015 - Accepted 1 February 2016 - First published online 10 March 2016)

\section{Abstract}

Both undernutrition and hypoxia exert a negative influence on both growth pattern and bone mechanical properties in developing rats. The present study explored the effects of chronic food restriction on both variables in growing rats exposed to simulated high-altitude hypoxia. Male rats ( $n$ 80) aged $28 \mathrm{~d}$ were divided into normoxic $(\mathrm{Nx})$ and hypoxic (Hx) groups. Hx rats were exposed to hypobaric air $(380 \mathrm{mmHg}$ ) in decompression chambers. At $\mathrm{T}_{0}, \mathrm{Nx}$ and $\mathrm{Hx}$ rats were subdivided into four equal subgroups: normoxic control and hypoxic controls, and normoxic growth-restricted and hypoxic growth-restricted received $80 \%$ of the amount of food consumed freely by their respective controls for a 4 -week period. Half of these animals were studied at the end of this period $\left(\mathrm{T}_{4}\right)$. The remaining rats in each group continued under the same environmental conditions, but food was offered ad libitum to explore the type of catch-up growth during 8 weeks. Structural bone properties (strength and stiffness) were evaluated in the right femur midshaft by the mechanical three-point bending test; geometric properties (length, cross-sectional area, cortical mass, bending cross-sectional moment of inertia) and intrinsic properties of the bone tissue (elastic modulus) were measured or derived from appropriate equations. Bone mineralisation was assessed by ash measurement of the left femur. These data indicate that the growth-retarded effects of diminished food intake, induced either by food restriction or hypoxia-related inhibition of appetite, generated the formation of corresponding smaller bones in which subnormal structural and geometric properties were observed. However, they seemed to be appropriate to the body mass of the animals and suggest, therefore, that the bones were not osteopenic. When food restriction was imposed in Hx rats, the combined effects of both variables were additive, inducing a further reduction of bone mass and bone load-carrying capacity. In all cases, the mechanical properties of the mineralised tissue were unaffected. This and the capacity of the treated bones to undergone complete catch-up growth with full restoration of the biomechanical properties suggest that undernutrition, under either Nx or Hx conditions, does not affect bone behaviour because it remains appropriate to its mechanical functions.

\section{Key words: Undernutrition: Hypoxia: Bone quality: Biomechanics: Catch-up growth: Body mass}

The growing rat appears to follow an individual size-age trajectory to its body mass end point. This characteristic growth trajectory can be altered in rate and timing by exogenous modifiers. Two important non-genetic, environmental effects on the growth pattern are undernutrition ${ }^{(1-8)}$ and hypoxia (defined in terms of hypoxaemia, anaemia or increased Hb-oxygen affinity at sea level) ${ }^{(9-13)}$.

On the basis of clinical paediatric findings ${ }^{(13-15)}$, we have developed a nutritional stress model in rats (nutritional growthretarded rats): weanling male rats placed on a $20 \%$ restricted balanced diet for a 28 -d period closely resembled the suboptimal nutrition observed in children who consume inappropriate diets with insufficient total energy to sustain normal growth and weight gain $^{(16)}$. Rats maintained on this type of chronic suboptimal nutrition decreased their body mass growth rate, which was one of several described manifestations of nutritional inadequacy.
When undernutrition was prolonged until animals reached adulthood, the body mass reached a plateau that was significantly lower than that found in well-nourished age-matched rats.

Under hypobaric hypoxia, induced by exposure to simulated high altitudes (SHA) in hypobaric chambers, body mass growth rate is impaired in developing rats. As a consequence, body mass is reduced for the chronological age and sex. The effect of hypoxia on body mass growth rate has been associated with hypophagia because of reduced appetite ${ }^{(11,12,17,18)}$. It has also been suggested ${ }^{(19)}$ that body mass growth retardation during exposure to hypobaric hypoxia could be due to changes in a central control (set-point) with a mechanism for setting target body mass. A reduced body mass may be adaptive to high altitude by reducing oxygen needs ${ }^{(20)}$.

The above explanation indicates that increase in body mass is negatively affected by either an 'imposed' or a 'voluntary'

Abbreviations: CSA, cross-sectional area; Hx, hypoxic; HxC, hypoxic control; HxGR, hypoxic growth-restricted; Nx, normoxic; NxC, normoxic control; NxGR, normoxic growth-restricted; xCSMI, second moment of inertia of cortical bone area concerning anterior-posterior bending.

*Corresponding author: C. E. Bozzini, fax +54 114508 3958, email cebozi@fisio.odon.uba.ar 
restriction of food consumption, as occurs in both the normoxic growth-restricted (NxGR) rat and the hypoxic control (HxC) rat, respectively. Catch-up growth, defined as growth with a velocity above the statistical limits of normality for age during a defined period of time, which follows a period of impaired growth $^{(21)}$, was observed in both models ${ }^{(19,22)}$.

Both NxGR and HxC rats also have in common important changes in the mechanical properties of the appendicular skeleton. Biomechanical tests performed on femur diaphyseal shafts $^{(21,23)}$ revealed a lower than normal bone mass and changes in the geometric properties of the bone, without alterations in the material properties of the bone tissue. The studied bones, because of the subnormal strength and stiffness, were thus structurally incompetent for sex and age.

The present investigation was designed to evaluate the behaviour of body mass and the mechanical properties (structural, architectural and material) of bone in developing male rats showing a voluntary nutritional-restricted growth because of exposure to hypobaric hypoxia, in which a further restriction of food availability was superimposed in the same proportion used to develop a NxGR rat (hypoxic growthrestricted (HxGR) rat). The effects of two exogenous factors acting simultaneously on the body and bone developing trajectories could thus be analysed. In other words, the effects of suboptimal nutrition in high altitudes were experimentally evaluated. The presence of complete or incomplete catch-up growth in response to unrestricted food availability was estimated in both NxGR and HxGR rats (NxGRad and HxGRad, respectively) after the 4 -week period of food restriction.

\section{Methods}

\section{Experimental subjects}

Wistar male rats aged $28 \mathrm{~d}$ and weighing 63.42 (SE 0.35) g were used as experimental subjects. They were housed in stainlesssteel, wire-bottomed cages under a natural light-dark cycle in a temperature-controlled $\left(22-24^{\circ} \mathrm{C}\right)$ room. Animals were fed a standard diet (Purina chow) with the following composition (g/100 g): protein, 23.5; lipids, 7.09; fibre, 6.0; Ca, 1.3; P, 0.8; ash, 6.39; water, 7.96; and dextrin up to $100 \mathrm{~g}$.

\section{Experimental design}

At $\mathrm{T}_{0}$, rats were divided into equal groups of forty animals each, normoxic $(\mathrm{Nx})$ and hypoxic $(\mathrm{Hx})$. Nx rats were maintained at sea-level conditions $(760 \mathrm{mmHg}, 0 \mathrm{~m})$, whereas $\mathrm{Hx}$ rats were exposed to hypobaric air $(380 \mathrm{mmHg}, 5450 \mathrm{~m}$ ) during the entire experimental period, which lasted 12 weeks. This value of hypobaric air was chosen because it was found to induce a marked inhibition of growth rate in immature rats ${ }^{(23)}$. Hypoxia was induced by placing rats into simulated altitude chambers in which the desired air pressure was maintained using vacuum pumps and adjustable inflow valves. Exposure was intermittent (minimum 22-23 h/d) with a daily interruption to replace food and water, clean animal cages and perform experimental manoeuvres when necessary. Exposure was continuous from Friday to Monday of every week. As the exposure was performed in hypobaric chambers that do not reproduce completely the many stresses that animals and humans experience in high altitude (cold, ionising radiation, dry air, etc.) but only reduced atmospheric pressure, animals in this study were really exposed to SHA. At $\mathrm{T}_{0}$, both $\mathrm{Nx}$ and $\mathrm{Hx}$ groups of rats were subdivided into eight equal subgroups of ten animals each (see Table 1): (I)normoxic control (NxC1) rats with free access to food during the entire experimental period (12 weeks); (II) $\mathrm{NxC} 2$, idem anterior that were sacrificed at 4 weeks; (III) NxGR rats that received $80 \%$ of the amount of food consumed freely by $\mathrm{NxC} 2$ on the previous day, corrected by body weight (food intake in $\mathrm{g} / 100 \mathrm{~g}$ body weight/d); (IV) NxGRad, idem anterior with restriction of food intake during the first 4 weeks and intake ad libitum during the next 8 weeks; (V) HxC1 rats treated as $\mathrm{NxC1}$; (VI) $\mathrm{HxC} 2$ rats treated as $\mathrm{NxC2}$; (VII) $\mathrm{HxGR}, \mathrm{Hx}$ rats treated as $\mathrm{NxGR}$; and (VIII) HxGRad, Hx rats treated as NxGRad. Rats were euthanised by injecting ketamine $(0.1 \mathrm{ml} / 100 \mathrm{~g}$ body weight $)$ and xilasine $(0.02 \mathrm{ml})$. During the study period, body mass (recorded in a Mettler P600 scale every 2 d; Mettler Instrument Corporation) and body length (distance from the tip of the nose to base of tail, measured weekly) were registered periodically. At the end, final body mass and body length were established. Body weight was taken as representative of body mass. The value of the body weight registered at $5400 \mathrm{~m}$ will be 0.99066 of that measured at sea level because of the lower gravitational acceleration. The difference is negligible, which allows comparison of body weight values between Nx and Hx rats. Animals were then euthanised as described above. The femurs were removed, cleaned of adherent soft tissue, weighed in a Mettler scale and stored at $-20^{\circ} \mathrm{C}$ wrapped in gauze soaked with Ringer's solution in sealed plastic bags, as recommended by Turner \& Burr ${ }^{(24)}$.

\section{Biomechanical testing}

On the day of testing, each bone sample was thawed at room temperature for $4 \mathrm{~h}$ before analysis. To assess cortical bone mechanical properties, the right femur was subjected to a

Table 1. Experimental design*

\begin{tabular}{|c|c|c|c|c|c|}
\hline Group & & Food intake & $\mathrm{A} 1$ & Food intake & $\mathrm{A} 2$ \\
\hline Period... & $n$ & First 4 weeks & 4 weeks & Second 8 weeks & 12 weeks \\
\hline $\mathrm{NxC1}$ & 10 & Ad libitum & No & Ad libitum & Yes \\
\hline $\mathrm{NxC2}$ & 10 & Ad libitum & Yes & & \\
\hline NxGR & 10 & $80 \%$ & Yes & & \\
\hline NxGRad & 10 & $80 \%$ & No & Ad libitum & Yes \\
\hline $\mathrm{HxC1}$ & 10 & Ad libitum & No & Ad libitum & Yes \\
\hline $\mathrm{H} \times \mathrm{C} 2$ & 10 & Ad libitum & Yes & & \\
\hline HxGR & 10 & $80 \%$ & Yes & & \\
\hline HxGRad & 10 & $80 \%$ & No & Ad libitum & Yes \\
\hline
\end{tabular}

A1 and A2, Autopsy 1 and autopsy 2 were performed at 4 and 12 weeks of the experimental period, respectively; $\mathrm{NxC} 1$ and $\mathrm{NxC2}$, normoxic control rats; NxGR, normoxic growth-restricted rats; NxGRad, normoxic rats with food restriction during the first 4-week period, and consumption ad libitum during the second 8-week period; $\mathrm{HxC1}$ and $\mathrm{HxC2}$, hypoxic control rats; $\mathrm{HxGR}$, hypoxic growth-restricted rats; HxGRad, hypoxic rats with food restriction during the first 4-week period, and consumption ad libitum during the second 8-week period.

* The experimental period lasted for 12 week, divided into the first (4 weeks) and the second (8 weeks) periods. 
three-point bending mechanical test ${ }^{(24,25)}$, which combines compression and tension. Each bone was placed horizontally with the anterior side facing down on two transverse supports and centred along its length. The assayed bone portion was always within two-fifth of the total bone length. This condition rendered the method suitable for comparative purposes ${ }^{(26)}$. Load was applied perpendicularly at the medial aspect of the long axis of the bone until fracture. The test machine (Instron model 4442; Instron Corp.) was operated in stroke control at a constant rate of $5 \mathrm{~mm} / \mathrm{min}$, which is useful for describing the static properties of the bone structure. The resulting load/ deformation (W/d) curves showing both the elastic (Hookean behaviour) and the plastic (non-Hookean behaviour) phases separated by the yielding point enabled computerised determination of the main structural mechanical properties of bone shafts as beams ${ }^{(24)}$, which essentially measures the resistance to both deformation (stiffness) and fracture (strength) as well as the ability to absorb energy by deforming. The structural properties are those corresponding to the whole bone as an organ and are derived from the W/d curve. They are as follows: (i) load at the yielding point or elastic limit (Wy represents the end point of the elastic deformation of the bone (yielding point) and defines a threshold about which unrecoverable permanent deformation occurs (plastic deformation), marking the initiation of damage accumulation with the appearance of microcracks that occur on the periosteal surface of the bone subjected to tension; it is a measure of the bone strength); (ii) structural stiffness or bone rigidity (Wydy, represents the slope of the elastic phase of the $\mathrm{W} / \mathrm{d}$ curve and is a measure of the resistance of the bone to deformation, or rigidity); and (iii) structural strength (Wf, represents the value of the load at fracture and expresses directly the resistance of the whole bone to fracture, incorporating both the elastic and the plastic behaviours). Geometric or architectural properties represent the bone design characteristics; they are (i) Bone length and diameters. Using an Isomet low-speed diamond saw (Buehler), the fracture section of each fractured femur was regularised to perform micromorphometrical determinations of the vertical (load direction) and horizontal (right angle to load direction) outer (vertical outer diameter (VOD), horizontal outer diameter (HOD)) and inner (vertical inner diameter (VID), horizontal inner diameter (HID)) diameters of the section. Measurements were taken directly using a stereomicroscope (Stemi DV4; Carl Zeiss Microimaging) with an accuracy of $\pm 0.001 \mathrm{~mm}$. The crosssectional area (CSA) was calculated by applying the following equation: $\mathrm{CSA}=\pi(\mathrm{VOD} \times \mathrm{HOD}) / 4$. CSA provides information on the bone size and is an important factor that influences the ability of a bone to resist a particular load. Therefore, larger the CSA, the smaller the amount of deformation induced by a load (ii). The cross-sectional cortical area (CtA) is the part of the CSA between the periosteal and endocortical layers that is mainly composed by compact bone. It can be taken as representative of the bone mass. It was calculated using the following equation: $\mathrm{CtA}=\pi(\mathrm{VOD} \times \mathrm{HOD}-\mathrm{VID} \times \mathrm{HID} / 4$. The medullar area is the part of the CSA within the endosteal layer and is mainly composed of trabecular bone and bone marrow. Trabecular bone is sparse at the femoral midshaft. The second moment of inertia of cortical bone area concerning anterior-posterior bending (xCSMI) was estimated by the following equation: $x \mathrm{CSMI}=\left(\pi\left(\mathrm{VOD}^{3} \times \mathrm{HOD}-\mathrm{VID}^{3} \times \mathrm{HID} / 64\right)\right)$; $\mathrm{xCSMI}$ captures both bone mass and distribution in the cross section. Larger the XCSMI, further the disposition of bone cortical mass from the internal-external $(x)$ axis of the cross-section. Bone material properties (mechanical properties of the mineralised tissue) were not directly determined by mechanical means but calculated from estimated structural and geometric properties. The Young's modulus of elasticity (E), which is indicative of the bone material stiffness, or intrinsic stiffness, was calculated using the following formula: $\mathrm{E}=\mathrm{WyL}^{3} / 48 \mathrm{dy}$. Ix (Wy $=$ load at the yielding point, $\mathrm{L}=$ distance between supports, $\mathrm{dy}=$ maximal elastic deflection, Ix = second moment of inertia of the crosssection in relation to the horizontal axis). Structural analyses of whole bones generally approximate effective material properties by assuming that the tissue is homogeneous. However, bone tissue is a heterogeneous material with properties that vary both temporally and spatially within the tissue microstructure. The values of tissue elastic modulus estimated from whole-bone structural tests are considerably lower than that expected based on nanoindentation results, and the effective material properties do not correlate with directly related measured material properties $^{(24)}$. However, data on material properties of bone obtained by structural analysis (present investigation) can be used for comparative purposes between control and experimental animals.

\section{Ashing of the specimens}

The left femur of each animal was ashed at $600^{\circ} \mathrm{C}$ in a muffle furnace for $18 \mathrm{~h}$ and ash weight was obtained. The tissue degree of mineralisation $(\alpha)$, which expresses the percentage of mineral substance in the dried bone, was calculated as the ratio of ash weight:dry bone weight.

Bone can be studied at several organisation levels. On the basis of the theory of continuous materials, bone hierarchy can be arranged into whole-bone, architectural, tissue, lamellar and ultrastructural levels ${ }^{(27)}$. The present investigation estimated mechanical properties of the entire bone as a structure, which incorporates the properties of the materials that compose the whole bone, as well as its internal and external geometry. The femur, a weight-bearing bone, was chosen as representative of the peripheral skeleton. The femoral mid-diaphysis in the rat is primarily composed of cortical tissue, whose primary function is to provide strength and support. Therefore, this study really evaluated the effects of treatments on cortical bone biomechanics.

\section{Statistical analysis}

Results are summarised as mean values with their standard errors and were considered statistically significant at the level of $P<0.05$. Comparisons between parameters were performed by one-way ANOVA and test of Student-Newman-Keuls using GraphPad Software (GraphPad Software Inc.).

\section{Ethics}

The experiment was conducted in accordance with the principles outlined in the National Institute of Health Guide for the 
Care and Management of Laboratory Animals, and was approved by the University of Buenos Aires Ethics Committee.

\section{Results}

Fig. 1 summarises the effects of treatments on both body mass and body length. The growth rate of body mass (Fig. 1(C)) was depressed by $38.5 \%$ in well-nourished rats exposed to hypobaric air during the $\mathrm{T}_{0}-\mathrm{T}_{28}$ period $(\mathrm{HxC})$ when compared with well-nourished $\mathrm{NxC}$ rats. As a consequence, body mass was $25 \cdot 1 \%$ less in $\mathrm{HxC}$ than in $\mathrm{NxC}$ animals at the end of the first 28-d period (Fig. 1(A)). Growth rates were significantly stunted by the imposed $20 \%$ food restriction. The parameter

(A)

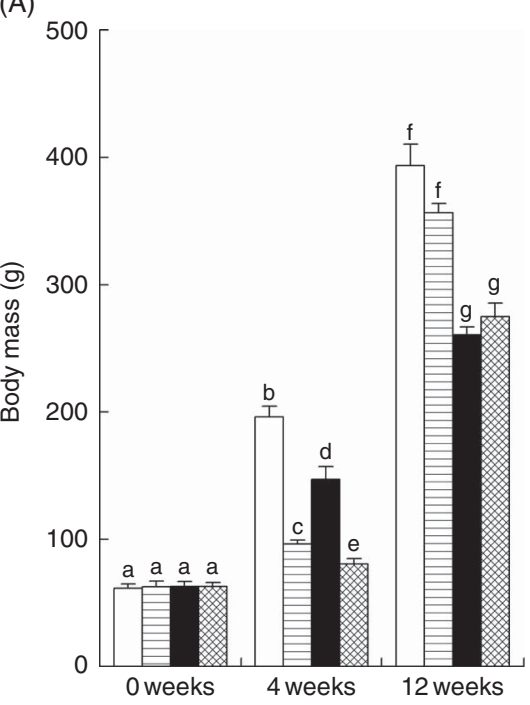

(C)

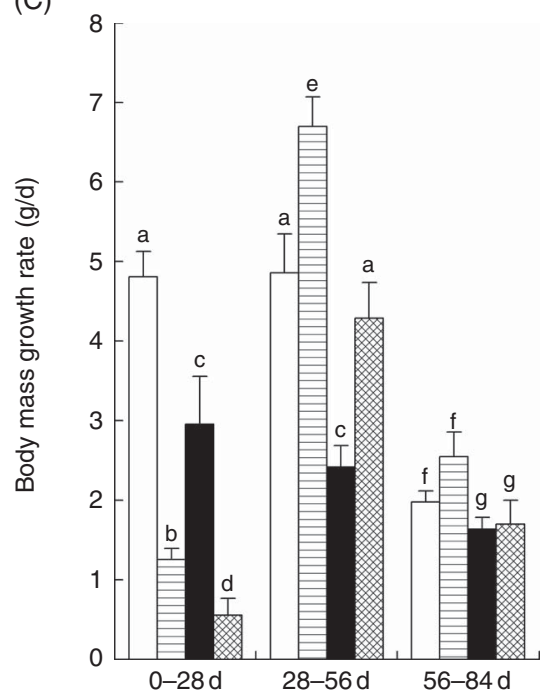

was 26.2 and $18.9 \%$ depressed in $\mathrm{Nx}$ and $\mathrm{Hx}$ rats, respectively, when compared with their well-nourished controls. In response to this depressed body mass growth rates, the resultant body mass was lowered by $50 \cdot 1$ and $46 \cdot 2 \%$ in NxGR and HxGR rats, respectively, compared with their relevant controls (Fig. 1(A)). During the initial part of the recovery from food restriction $\left(\mathrm{T}_{28}-\mathrm{T}_{56}\right)$, growth parameters increased significantly. Body mass growth rates were $37 \cdot 8$ and $77 \cdot 3 \%$ higher in NxGR and HxGR rats, respectively, when compared with their respective controls. No explanations are evident for this difference in efficiency of growth rate between NxGR and HxGR. During the final part of the recovery period $\left(\mathrm{T}_{56}-\mathrm{T}_{84}\right)$, growth rate declined in all groups in relation to the previous one, approaching control values. The increased velocity of body

(B)

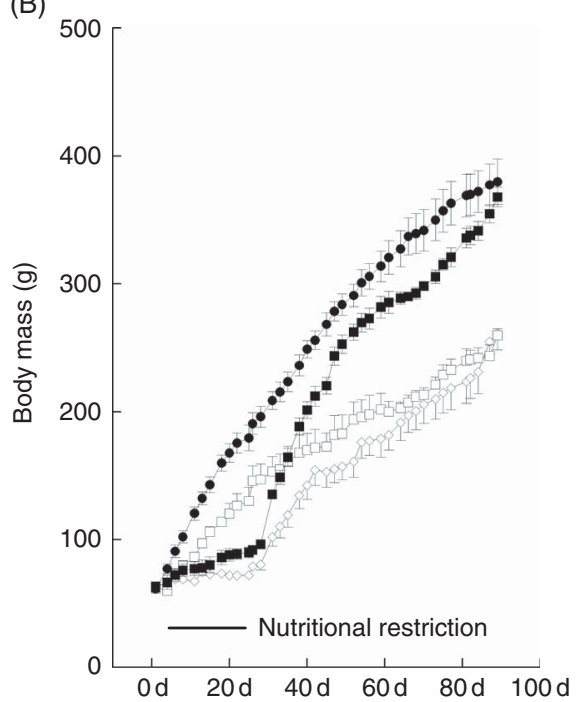

(D)

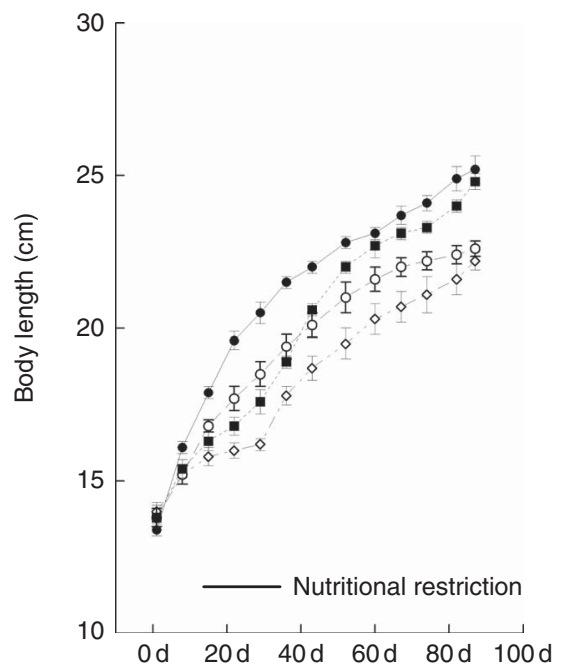

Fig. 1. Body mass, body mass growth rate and body length in normoxic, well-nourished rats $(\mathrm{NxC})$, normoxic growth-restricted rats (NxGR), hypoxic well-nourished rats $(\mathrm{HxC})$ and hypoxic growth-restricted rats ( $\mathrm{HxGR}$ ). (A) Values of the bone mass at the start of the experiment (0 weeks), at the end of the food-restriction period (4 weeks) and at the end of the recovery period, or final body mass (12 weeks). (B) Changes in body mass with time in the four studied groups. (C) The body mass growth rate, expressed in $\mathrm{g} / \mathrm{d}$, during the periods of food restriction (0-28 d), recovery (28-56 d) and final (58-84 d). (D) Changes in body length with time in the four studied groups. a,b,c,d,e,f,g Mean values within a column with unlike letters were significantly different. In Y/X curves, each value represents the mean values ( $n 10$ animals), with their

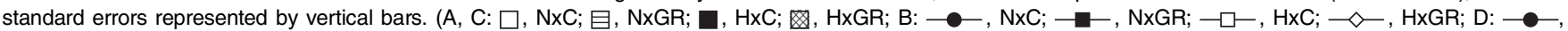
$\mathrm{NxC} ;-$ - NxGR; -..-.., HxC; - - - - , HxGR). 
mass growth during the recovery period in NxGR and HxGR animals determined that no significant differences were evident between NxC and NxGR, on the one hand, and HxC and HxGR, on the other hand, at the end of the experimental period (day 84). However, body mass at this time was significantly less in $\mathrm{Hx}$ than in $\mathrm{Nx}$ rats (approximately 30\%). As shown in Fig. 1(D), body length as a function of time showed a similar pattern of changes as that of body mass (Fig. 1(B))

Both femur weight and femur length were similarly and negatively affected by both food restriction (NxGR) and exposure to hypoxia (HxC) (Fig. 2(A) and (B)). Food restriction imposed on Hx rats (HxGR) further delayed the growth of both parameters. Both weight and length showed a complete type of catch-up $^{(28)}$ during the recovery phase. However, catch-up growth was complete in NxGR rats when compared with their $\mathrm{NxC}$, whereas catch-up growth was complete in HxGR rats when compared with their HxC. Thus, the subnormal weight and length of the femur that was observed in $\mathrm{Hx}$ rats during the growth phase in relation to $\mathrm{Nx}$ ones ${ }^{(23)}$ were observed again in the present experiment when determined at the end of the recovery period. The diaphyseal design indicators, evaluated in this study by the cortical bone area (Fig. 2(C)) and the xCSMI (Fig. 2(D)), and the load-bearing capacity of the diaphyseal shaft (load at yielding (Fig. 2(E)), load at fracture (Fig. 2(F)) and structural stiffness (Fig. 2(G)) were negatively affected by either hypoxia in well-nourished rats $(\mathrm{HxC})$ or food restriction in both NxGR and HxGR animals: hypoxia alone (HxC) and food restriction in normoxia (NxGR) affected cortical area in the same proportion. The combined effects of hypoxia and food restriction (HxGR) induced the maximal negative effects on all variables studied. Both geometrical and structural variables grossly paralleled changes in body weight. As an example, diaphyseal strength (fracture load) was found to be positively and linearly correlated with body weight, considering the data from all groups as a whole $\left(r 0.9282 ; r^{2} 0.8616 ; P<0.0001\right)$. All affected parameters showed complete catch-up at the end of the period in which animals had free access to food. Neither hypoxia nor food restriction affected either the Young's modulus or the degree of mineralisation (Fig. 2(H) and Fig. 2(I), respectively).

\section{Discussion}

This study examined the (1) the pattern of growth of body mass in well-nourished male rats during the 84-d post-weaning period, maintained at either sea-level conditions or high altitude chambers at a simulated altitude equivalent to $5400 \mathrm{~m}$ ( $0.5 \mathrm{~atm}$ ) (chronic hypoxia); (2) the effects on the same variable of an imposed $20 \%$ reduction in food intake during the first $28 \mathrm{~d}$ in both groups of animals and the type of catch-up occurring after removal of food restriction during the remaining $56 \mathrm{~d}$ of the experimental period; and (3) the behaviour of the biomechanical properties of the femur shaft under these experimental conditions.

Heredity is the primary determinant of physical development. Genes code for all the functional proteins the body needs to grow and mature, and every animal is born with a unique genetic map. However, other factors influence growth and development, such as sex, nutrition, hormones, environment, etc.
In the present investigation, we have confirmed the adverse effects on the growth trajectory of growing rats of two exogenous modifiers, both a $20 \%$ nutritional restriction ${ }^{(21,22)}$ and exposure to an hypobaric environment ${ }^{(11,19,23)}$. Nutritional restriction impairs body mass growth mainly by lowering plasma insulin-like growth factor $\mathrm{I}^{(29,30)}$, whereas hypobaric hypoxia affects growth by creating nutritional restriction by inducing hypophagia in response to decreased appetite ${ }^{(11,12,17-19)}$. Thus, both conditions appear to have in common a similar reason to impair growth. The negative effect of exposure to environmental hypoxia on body mass growth has been shown to be positively correlated to the degree of altitude exposure ${ }^{(23)}$ and, under these conditions, to the amount of food consumed ${ }^{(12)}$. Thus, the negative influence of altitude hypoxia on body mass growth might be seen as a suboptimal nutrition effect.

In the present study, both food restriction and hypoxia, acting separately, induced significant growth deceleration that was responsible for the reduction in body mass observed in animals under these treatments at the end of a 4-week period (Fig. 1(B), NxGR and HxC). When food restriction was imposed in $\mathrm{Hx}$ rats, their body masses were further decreased (Fig. 1(B), HxC $v$. HxGR) because of the adverse effect on growth rate evoked by the imposed food restriction (20\%) plus the voluntary restriction of food intake induced by hypoxia. During the recovery period, in which food was offered ad libitum to all restricted rats (NxGRad and HxGRad), it was observed that rats markedly increased their food intake so as to permit catch-up growth. Catch-up growth was complete (the growth deficit was swiftly eliminated when restriction ceased) in both groups of nutritionrestricted rats - Nx and $\mathrm{Hx}$ - with the following characteristics: Nx rats previously stunted by undernutrition attained a final body mass that was within the growth trajectory of $\mathrm{Nx}$ rats, whereas Hx rats similarly treated reached a final body mass that was within the growth trajectory of $\mathrm{Hx}$ rats. The former attained a 'Nx' body mass, whereas the latter attained a ' $\mathrm{Hx}$ ' body mass. These data thus provide support to our previously reported hypothesis ${ }^{(19)}$ that body mass might be negatively affected by hypoxia as a result of a reset of a putative central set-point with a mechanism for setting target mass size of the body, which operates by depressing appetite, probably in response to hypoxia-induced hypometabolism ${ }^{(20)}$. However, hypometabolism in mammals is likely to be an immediate, emergency-type response to hypoxia, not desirable in chronic conditions, whereas hypophagia is a long-lasting effect of hypoxia. It is noteworthy that gestational hypoxia and early postnatal altitude hypoxia do not modify the body mass growth trajectory up to adulthood in rats when the offspring are raised under $\mathrm{Nx}$ conditions, and thus do not create lifelong consequences on body mass development ${ }^{(31)}$ (Fig. 3 and Fig. 4).

Undernutrition (in response to either food restriction or exposure to hypoxia), which mainly affects protein and energy availability, affects bone biomechanical properties ${ }^{(21,29,32-36)}$ in growing rats by adapting the growth of the bone mass to that of the body mass. Bone mechanical quality of a whole bone (structural properties) depends on the integration of the 'mechanical quality of the mineralised tissue' (material stiffness mainly associated with collagen mineralisation) and the 'architectural quality of the structural bone design' 
(A)

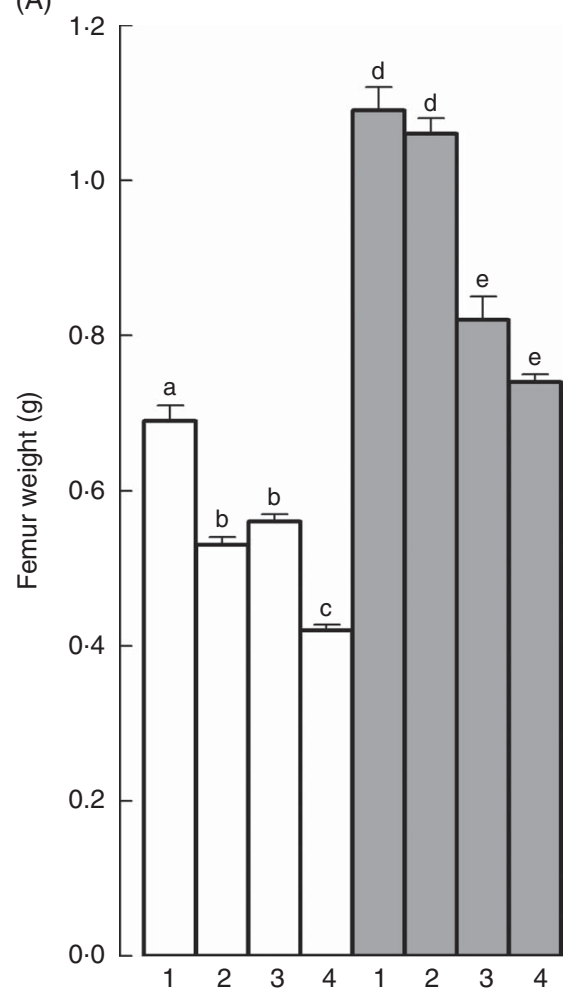

(C)

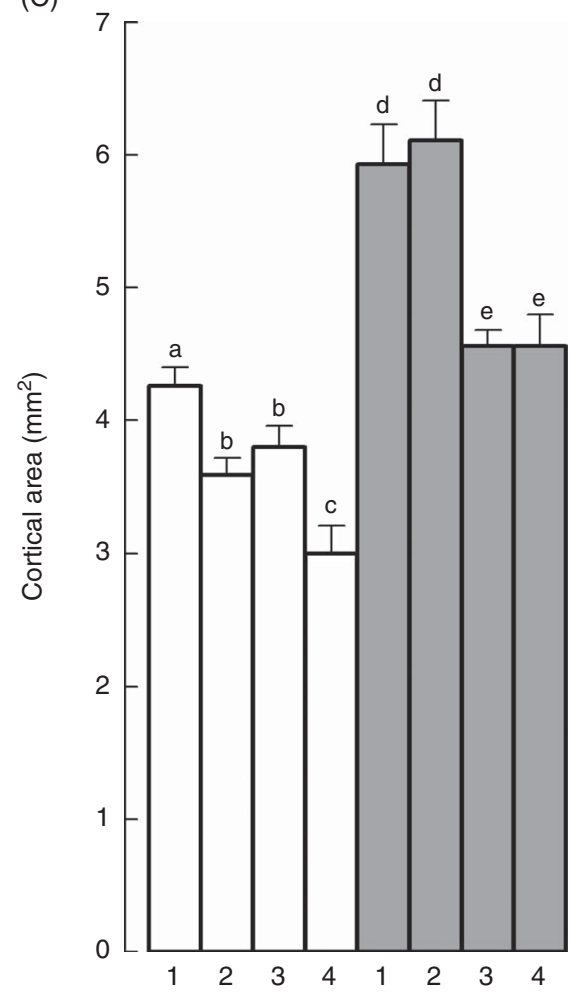

(B)

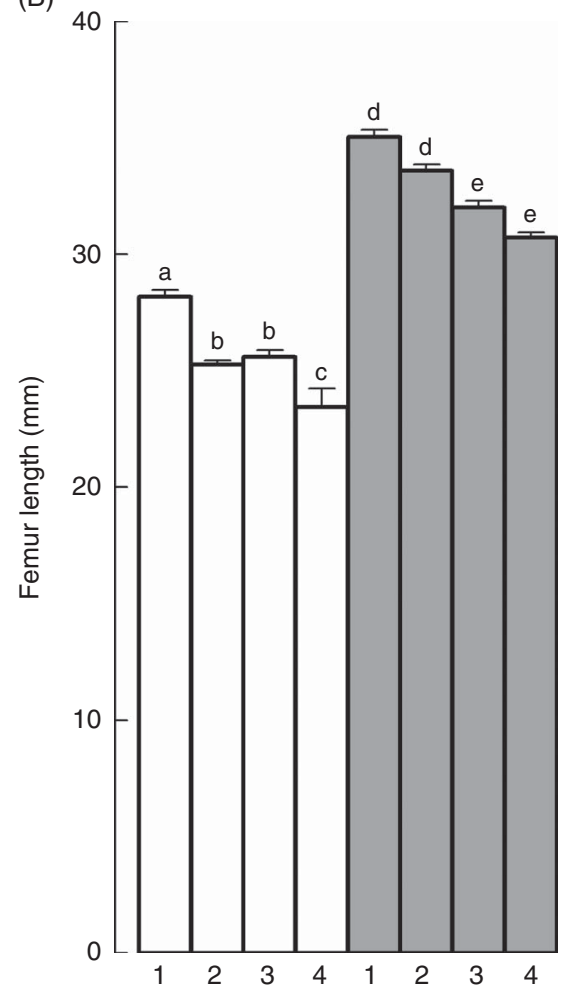

(D)

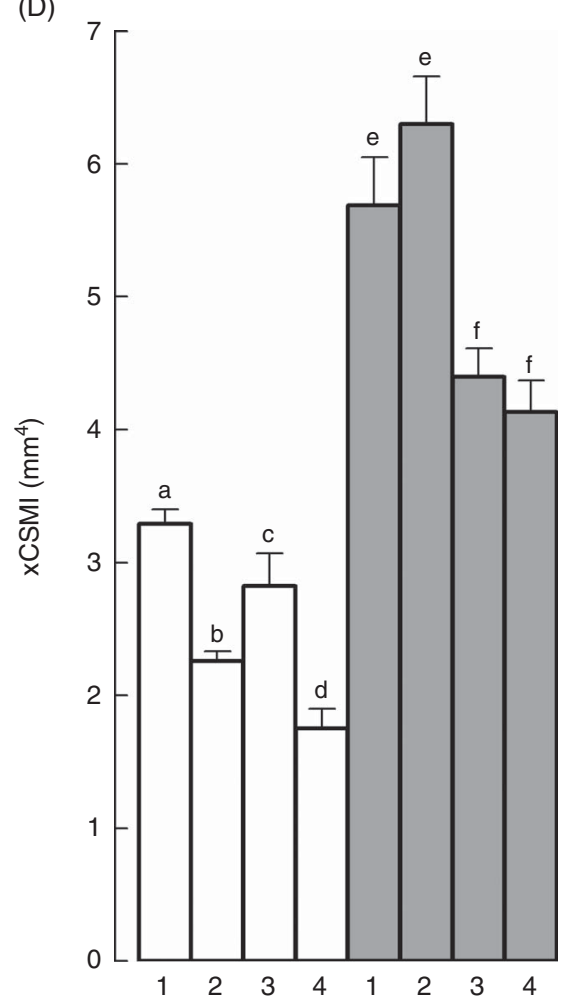

Fig. 2. Femur weight (A), femur length (B), cortical area (C) and cross-sectional moment of inertia (D) obtained from rats sacrificed at 4 weeks (restriction period, $\square$, autopsy 1 ) or 12 weeks (recovery period, $\square$, autopsy 2 ). The groups are as follows: $1=$ normoxic control; $2=$ normoxic growth restricted; $3=$ hypoxic control; and $4=$ hypoxic growth restricted. Values are means ( $n 10$ animals), with standard errors represented by vertical bars. ${ }^{a, b, c, d, e, f}$ Mean values within a column with unlike letters were significantly different. xCSMI, second moment of inertia of cortical bone area concerning anterior-posterior bending. 
(A)

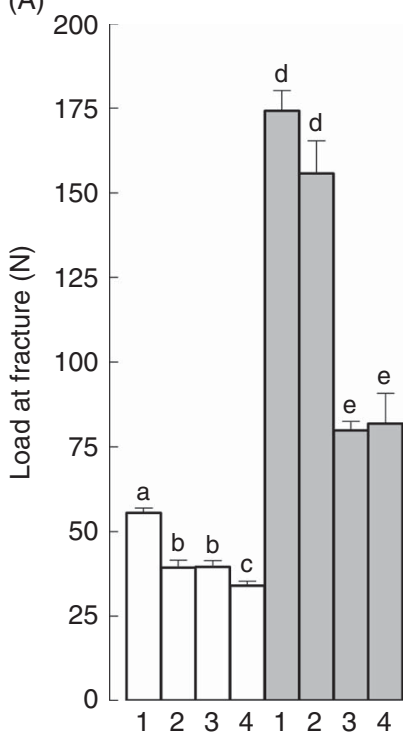

(B)

400

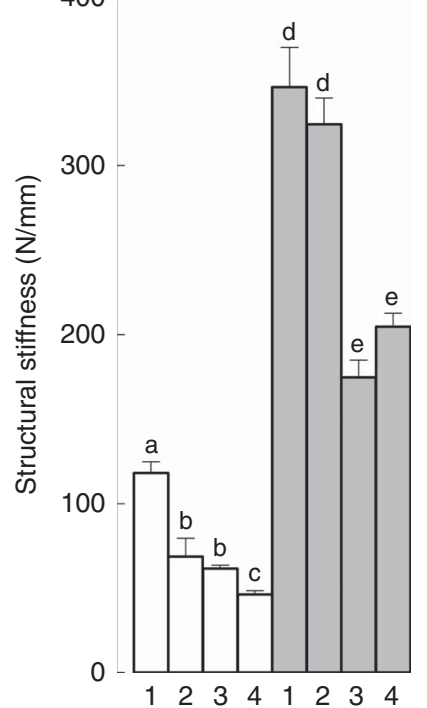

(C)

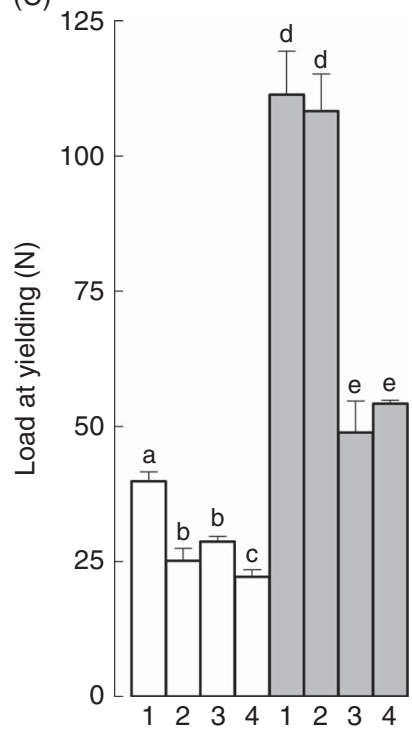

Fig. 3. Load at fracture (A), structural stiffness (B) and load at yielding (C) of the femur obtained from rats sacrificed at 4 weeks (restriction period, $\square$, autopsy 1 ) or 12 weeks (recovery period, $\square$, autopsy 2). The groups are as follows: $1=$ normoxic control; $2=$ normoxic growth restricted; $3=$ hypoxic control; and $4=$ hypoxic growth restricted. Values are means ( $n 10$ animals), with standard errors represented by vertical bars. ${ }^{a, b, c, d, e}$ Mean values within a column with unlike letters were significantly different.

(A)

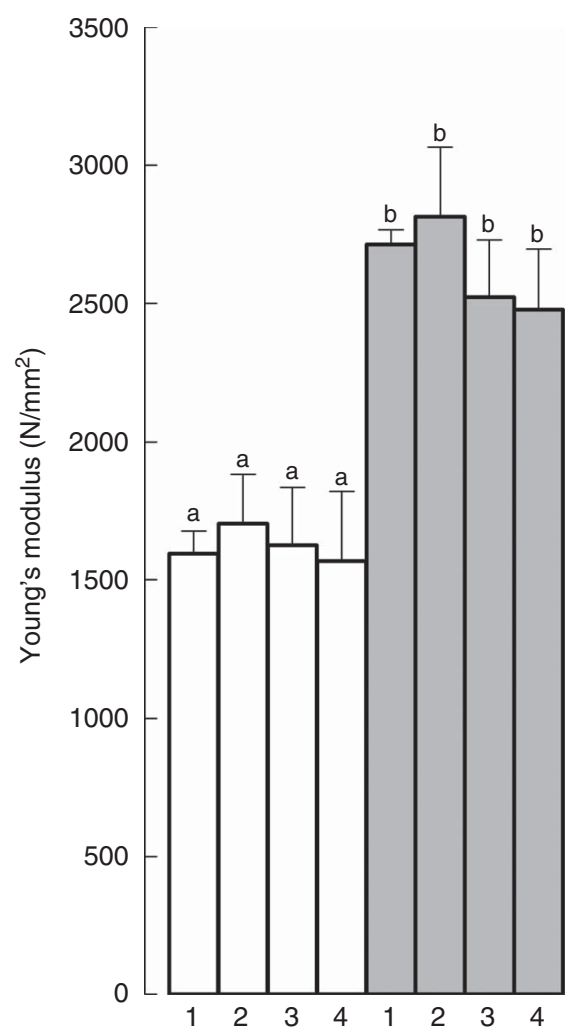

(B)

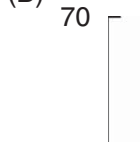

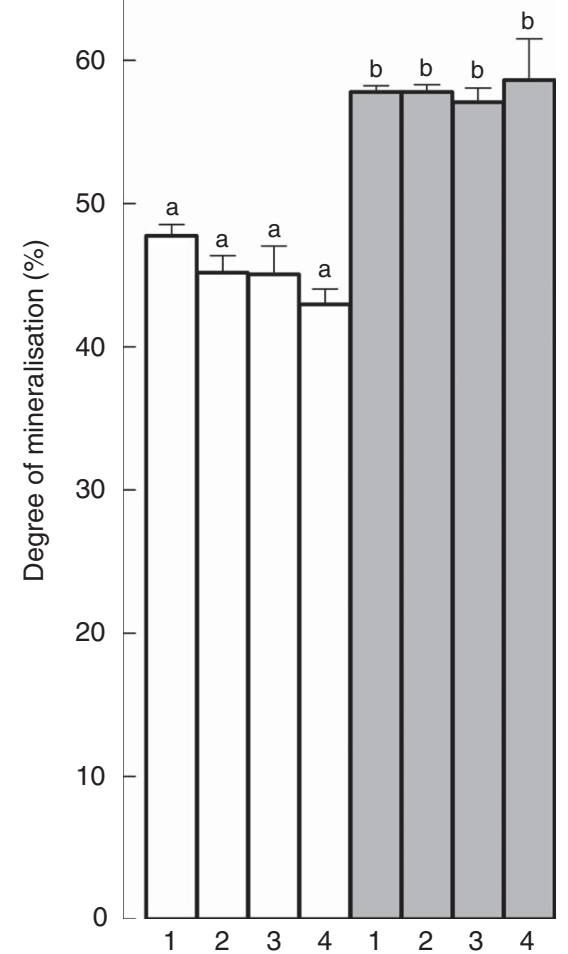

Fig. 4. Young's modulus of elasticity (A) and degree of mineralisation (B) of the right and left femur, respectively, obtained from rats sacrificed at 4 weeks (restriction period, $\square$, autopsy 1 ) or 12 weeks (recovery period, $\square$, autopsy 2). The groups are as follows: $1=$ normoxic control; $2=$ normoxic growth restricted; $3=$ hypoxic control; and 4 =hypoxic growth restricted. Values are means ( $n 10$ animals), with standard errors represented by vertical bars of ten animals each. ${ }^{\text {a,b }}$ Mean values within a column with unlike letters were significantly different.

(shape, size, architectural distribution of mineralised tissue) $)^{(37)}$. In general, the mechanical quality of the mineralised tissue is not affected by undernutrition, as confirmed again in the present study by the findings of normality of the elastic modulus in the two models of experimental rats. However, the femurs of the undernourished rats, either $\mathrm{Nx}$ or $\mathrm{Hx}$, were weaker than that 
of the normal ones, as shown by the correlative diminution in resistance to fracture and structural stiffness of the diaphyseal shafts. In addition, the weight and length of the femur were undoubtedly affected by growth retardation in undernourished rats. The differences in CSA, the cortical bone area and the xCSMI indicate that the size of the bone, in terms of the middiaphyseal cross section, was significantly and negatively affected. Body mass is one of the important factors that influence bone ability to resist stress. The high positive linear correlation between the load at fracture of the femur and the body mass of animals in this study suggests that bone mass, and consequently the structural bone strength, increased following the normal proportionality with body mass in restricted rats. Therefore, the clear differences in strength and stiffness of femoral beams seemed to be the result of an induced subnormal gain in bone structural properties as a consequence of a correlative subnormal gain in bone growth and mass, but not in bone material properties.

In summary, the results of the three-point bending test used in the present experiment to evaluate whole-bone mechanical properties indicate that the growth-retarded effects of reduced food intake, induced either by food restriction or hypoxia-related inhibition of appetite, generated the formation of corresponding smaller bones in which subnormal structural and geometric properties were observed. However, they seem to be appropriate to the body mass of the animals and suggest, therefore, that the bones are not osteopenic. When food restriction was imposed to Hx rats, the combined effects of both variables were additive, inducing a further reduction in bone mass and load-carrying capacity of bones. In all cases, the mechanical properties of the mineralised tissue were unaffected. This and the capacity of the treated bones to undergo complete catch-up growth with restoration of their biomechanical properties suggest that undernutrition, under either $\mathrm{Nx}$ or $\mathrm{Hx}$ conditions, does not affect behaviour of bones because they remain appropriate to their mechanical functions.

\section{Acknowledgements}

The present investigation was supported by research grants from the University of Buenos Aires (P. B., PID 200201301001100BA and R. M. A., PID 20020130100126BA) and National Research Council (CONICET, C. E. B., PIP 11220130100479 CO). R. M. A. and C. E. B. are Career Investigators from CONICET.

P. B., R. M. A. and C. E. B. are the Chiefs of the Laboratory of Nutrition, of Bone Biology and of Hypoxia, respectively, of the Department of Physiology. They, along with C. L. and C. B., joined to design the study and analyse data. G. C., P. P. and A. A. R. are Doctoral fellows who carried out the study. C. E. B. wrote the article, which was discussed with the other members of the group in a seminar before its acceptance.

The authors declare that there are no conflicts of interest.

\section{References}

1. Lee M \& Mayers GS (1979) The effect of protein energy malnutrition on appositional bone growth in the rat. Experientia 35, 824-825.
2. Nakamoto T \& Miller SA (1979) The effect of protein-energy malnutrition on the development of bones in new born rats. J Nutr 109, 1469-1476.

3. Galler JR \& Proper KJ (1981) The effect of protein deficiency on weight gain and body composition in the developing rat. Nutr Rep Int 24, 885-892.

4. Glick PL \& Rower DJ (1981) Effect of chronic protein deficiency on skeletal development of young rats. Calcif Tissue Int 33, 223-231.

5. Mercer LP, Watson DF \& Ramlet JS (1981) Control of food intake in the rat by dietary protein concentration. J Nutr $\mathbf{1 1 1}$, 1117-1123.

6. Burns RA, Lefaive RH \& Milner JA (1982) Effect of dietary protein quantity and quality on the growth of dogs and rats. J Nutr 112, 1843-1853.

7. Barceló AC, Alippi RM, Pucciarelli HM, et al. (1983) Growth of the functional components of the rat skull in protein deficiency. Acta Physiol Lat Am 33, 85-87.

8. Miller JP \& German RZ (1999) Protein malnutrition affects the growth trajectories of the craniofacial skeleton in rats. J Nutr 129, 2061-2069.

9. Timiras PS, Krum AA \& Pace N (1957) Body and organ weights of rats during acclimatization to an altitude of 12470 feet. Am J Physiol 191, 598-604.

10. Delaquierre-Richardson L, Forbes S \& Valdivia E (1965) Effect of simulated high altitude on the growth rate of albino guinea pigs. J Appl Physiol 20, 1022-1025.

11. Alippi RM, Barceló AC, Río ME, et al. (1983) Growth retardation in the early developing rat exposed to continuous hypobaric hypoxia. Acta Physiol Lat Am 33, 1-5.

12. Elia R, Elgoyhen AB, Bugallo G, et al. (1985) Effect of exposure to reduced atmospheric pressure on body weight, food intake and body composition of growing rats. Acta Physiol Lat Am 35, 311-318.

13. Schwartz JE, Kovach A, Meyer J, et al. (1988) Brief, intermittent hypoxia restricts foetal growth in SpragueDawley rats. Biol Neonate 73, 313-319.

14. Lisshitz F \& Moses N (1988) Nutritional dwarfing: growth, dieting and fear of obesity. J Am Coll Nutr 7, 367-376.

15. Lifshitz F \& Moses N (1989) Growth failure: a complication of dietary treatment of hypercholesterolemia. Am J Dis Child 143, 537-542.

16. Kaplan RM \& Toshima MT (1999) Does reduced fat diet cause retardation in child growth? Prev Med 21, 33-52.

17. Westerterp-Plantenga MS, Westerterp KR, Rubbens $\mathrm{N}$, et al. (1999) Appetite at high altitude, Operation Everest III (Comex-97): a simulated ascent of Mount Everest. J Appl Physiol 87, 391-399.

18. Morel OE, Aubert R, Richalet JP, et al. (2005) Simulated high altitude decreases protein intake and lean body mass gain in rats. Physiol Behav 86, 145-153.

19. Bozzini CE, Lezón CE, Norese MF, et al. (2005) Evidence from catch-up growth and hoarding behaviour of rats that exposure to hypobaric air lowers the body mass set point. Growth Dev Aging 69, 81-88.

20. Mortola JP. (1993) Hypoxic metabolism in mammals. News Physiol Sci 8, 79-82.

21. Boyer PM, Campagnucci GE, Olivera MI, et al. (2005) Bone status in an animal model of chronic sub-optimal nutrition: a morphometric, densitometric and mechanical study. Br J Nutr 93, 663-669.

22. Friedman SM, Rodríguez PN, Olivera MI, et al. (1998) Enanismo por desnutrición: cronodinamia de los procesos metabólicos en ratas (Nutritional dwarfing: cronodynamic of the metabolic processes in rats). Medicina (B. Aires) 58, 282-286. 
23. Bozzini C, Champin GM, Alippi RM, et al. (2013) Static biomechanics in bone of growing rats exposed chronically to simulated high altitude. High Alt Med Biol 14, 367-374.

24. Turner CH \& Burr DB (1993) Basic biomechanics measurements of bone: a tutorial. Bone 14, 595-608.

25. Hogan HA, Groves JA \& Simpson HW (1999) Long-term alcohol consumption in the rat affects cross-sectional geometry and bone tissue material properties. Alcohol Clin Exp Res 23, 1825-1833.

26. Ferretti JL, Capozza RF, Mondelo M, et al. (1993) Interrelationship between densitometric, geometric and mechanical properties of rat femur. Inferences concerning mechanical regulation of bone modeling. J Bone Miner Res $\mathbf{8}, 1389-1395$.

27. Liebschner MAK (2004) Biomechanical considerations of animal models in tissue engineering of bone. Biomaterials $\mathbf{2 5}$, $1697-1714$

28. Boersma B \& Wit JM (1997) Catch-up growth. Endocrine Rev 18, 646-661.

29. Bourrin S, Ammann P, Bonjour JP, et al. (2000) Dietary protein restriction lowers plasma insulin-like plasma growth factor (IGF-I), impairs cortical bone formation, and induces osteoblastic resistance to IGF-I in adult female rats. Endocrinology 141, 3149-3155.

30. Friedman SM, Gamba CA, Boyer PM, et al. (2001) Growth deceleration and bone metabolism in nutritional dwarfing rats. Int J Food Sci Nutr 52, 225-233.
31. Bozzini CE, Champin GM, Bozzini C, et al. (2014) Gestational and early postnatal exposure to simulated high altitude does not modify postnatal body mass growth trajectory in the rat. High Alt Med Biol 15, 418-421.

32. Ferretti JL, Tessaro RD, Delgado TJ, et al. (1988) Biomechanical performance of diaphyseal shafts and bone tissue of femurs from protein-restricted rats. Bone Miner $\mathbf{4}$, 329-339.

33. Ferretti JL, Capozza R, Cointry G, et al. (1991) Additive effects of dietary protein and energy deficiencies on diaphysis and bone tissue of rat femurs as determined by bending tests. Acta Physiol Pharmacol Ther Latinoam 41, 253-262.

34. Bozzini C, Bozzini CE \& Alippi RM (2012) Biomechanical properties of femoral shafts and bone tissue in proteinmalnourished rats from weaning to adulthood. Comp Clin Pathol 21, 1159-1165.

35. Alippi RM, Picasso E, Huygens P, et al. (2012) Growthdependent effects of dietary protein concentration and quality on the biomechanical properties of the diaphyseal rat femur. Endocrinol Nutr 59, 35-43.

36. Bozzini C, Picasso EO, Champin GE, et al. (2013) Mechanical testing at the whole-bone level of the femur in immature rats stunted by cornstarch consumption. Food Funct 4, 1543-1551.

37. Ferretti JL (1997) Biomechanical properties of bone. In Bone Densitometry and Osteoporosis, pp. 143-161 [HK Genant, G Guglielmi and M Jergas, editors]. Berlin: Springer. 